\title{
Editorial
}

\section{Existence and Asymptotic Behaviour of Solutions of Differential and Integral Equations in Some Function Spaces}

\author{
Józef Banaś, ${ }^{1}$ Mohamed Abdalla Darwish, ${ }^{2}$ Leszek Olszowy, ${ }^{1}$ and Kishin Sadarangani ${ }^{3}$ \\ ${ }^{1}$ Department of Mathematics, Rzeszów University of Technology, Aleja Powstańców Warszawy 8, 35-959 Rzeszów, Poland \\ ${ }^{2}$ Mathematics Department, Science Faculty for Girls, King Abdulaziz University, Jeddah 21589, Saudi Arabia \\ ${ }^{3}$ Departamento de Matemáticas, Universidad de Las Palmas de Gran Canaria, Campus de Tafira Baja, \\ 35017 Las Palmas de Gran Canaria, Spain \\ Correspondence should be addressed to Józef Banaś; jbanas@prz.edu.pl
}

Received 4 December 2014; Accepted 4 December 2014

Copyright (C) 2015 Józef Banaś et al. This is an open access article distributed under the Creative Commons Attribution License, which permits unrestricted use, distribution, and reproduction in any medium, provided the original work is properly cited.

Differential, integral, and functional integral equations play very significant role in describing of several events occurring in real world. Obviously, in the theory of the mentioned equations we are interested, first of all, in establishing conditions guaranteeing the existence of solutions of those equations in various function spaces and miscellaneous function classes. This direction of investigations is closely related to numerous questions of qualitative theory of differential and integral equations. For example, if we are looking for solutions of those equations defined on the real half-axis, then we are interested in the asymptotic behaviour of solutions of equations in question. More precisely, we are searching conditions imposed on functions involved in considered equations which ensure the attractivity (local and global) and the stability or asymptotic stability of solutions of the investigated differential or integral equations. Moreover, we can also consider the solvability of those equations in the class of periodic or almost periodic functions.

Apart from the above indicated questions concerning the theory of differential and integral equations, there are also some other important problems being the object of investigations in the theory of those equations. For example, we can consider iterative methods concerning the solvability of differential and integral equations, the continuous dependence of solutions with respect to parameters, or the uniqueness of solutions. Obviously, apart from those classical problems we can also investigate several ones connected with the theory of the mentioned equations, such as the construction of Green functions for boundary value problems, semigroups of operators associated with various differential equations, and others.
The presented special issue is strongly connected with various aspects of the theory of differential and integral equations which were mentioned above. We focus mainly on the existence of solutions of those equations in various function spaces since the choice of a suitable function space enables us to obtain appropriate results connected with the method of investigations.

Now we are going to describe briefly the results located in the papers covering this special issue.

In the paper authored by J. C. Mena et al., an existence result for a quadratic integral equation with supremum is obtained with help of the classical Schauder fixed point theorem. The investigations of the paper are conducted in the space of functions satisfying the Hölder condition. Using a suitable sufficient condition for relative compactness in the mentioned function space the authors proved their existence result assuming some natural conditions.

S. Dudek and L. Olszowy investigated, in their paper that is published in this special issue, the existence and continuous dependence of solutions of a nonlinear quadratic Volterra integral equation upon a parameter. In the considerations they applied the technique associated with measures of noncompactness in the Fréchet space of functions being continuous on the real half-axis. The mentioned result concerning the continuous dependence of solutions of the integral equation in question is expressed in terms of the Hausdorff distance.

The paper of Y. Wang and X. Yi studies a Liénard type of $p$-Laplace equation with a deviating argument. Using Manasevich-Mawhin continuation theorem the authors obtained a few criteria ensuring the existence and uniqueness of 
a periodic solution of the discussed equation. The assumptions imposed in the paper require a few technicalities and estimates.

Y. Wei studied in his paper the existence and uniqueness of a periodic solution of the second order ordinary differential equation. Assuming some estimates on the first order partial derivatives of the right-hand side of considered differential equation and applying the classical Schauder fixed point principle, a result on the existence and uniqueness of a solution of that differential equation is derived.

The paper of I. Bachar and H. Mâagli is devoted to the study of the solvability of a superlinear fourth order ordinary differential equation with boundary conditions. The authors used a method based on the estimates of the Green function associated with the considered problem. It is shown that under appropriate assumptions the problem in question has a unique positive solution.

J. Benameur and M. Blel considered the asymptotic behaviour of solutions of a surface quasigeostrophic equation. It is shown that if initial data are small enough then solutions of the mentioned equation have the exponential growth with respect to the time. The considerations of the paper are conducted in homogeneous Sobolev spaces.

In the paper of $\mathrm{H}$. Wu et al. the transport equation with partly smooth boundary conditions arising in growing cell population is studied. The considerations are situated in the space $L_{p}$. It is shown that the transport operator associated with the considered equation generates a $C_{0}$ semigroup and the ninth order remainder of the Dyson-Phillips expansion of that semigroup is compact, among others.

The method of a generalized Green function is applied in the paper of X. Han and J. Huang. Due to that method the solvability result for the second order difference equation with boundary conditions is obtained. The paper has a computational character and exploits the tools of the theory of difference equations.

The paper of E. M. A. Hilal and T. M. Elzaki studies solutions of nonlinear partial differential equations using a Laplace variational iteration method, Laplace transforms, and convolution integral. Several examples illustrate the efficiency of the method described above. In those examples the authors consider both linear and nonlinear partial differential equations together with suitable boundary conditions.

We hope that the results contained in this special issue will create the inspiration for researchers working in the theory of differential, integral, and functional equations in miscellaneous function spaces.

\section{Acknowledgment}

The guest editors of this special issue would like to express their gratitude to the authors of all papers submitted for consideration. 


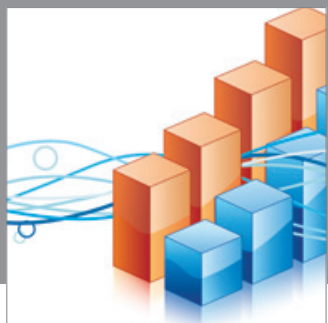

Advances in

Operations Research

mansans

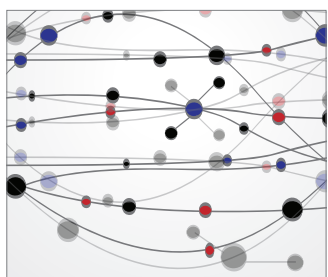

The Scientific World Journal
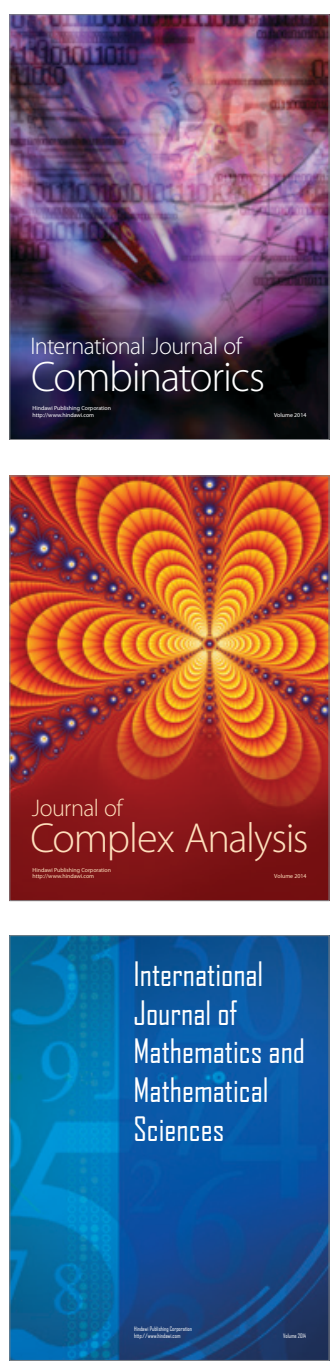
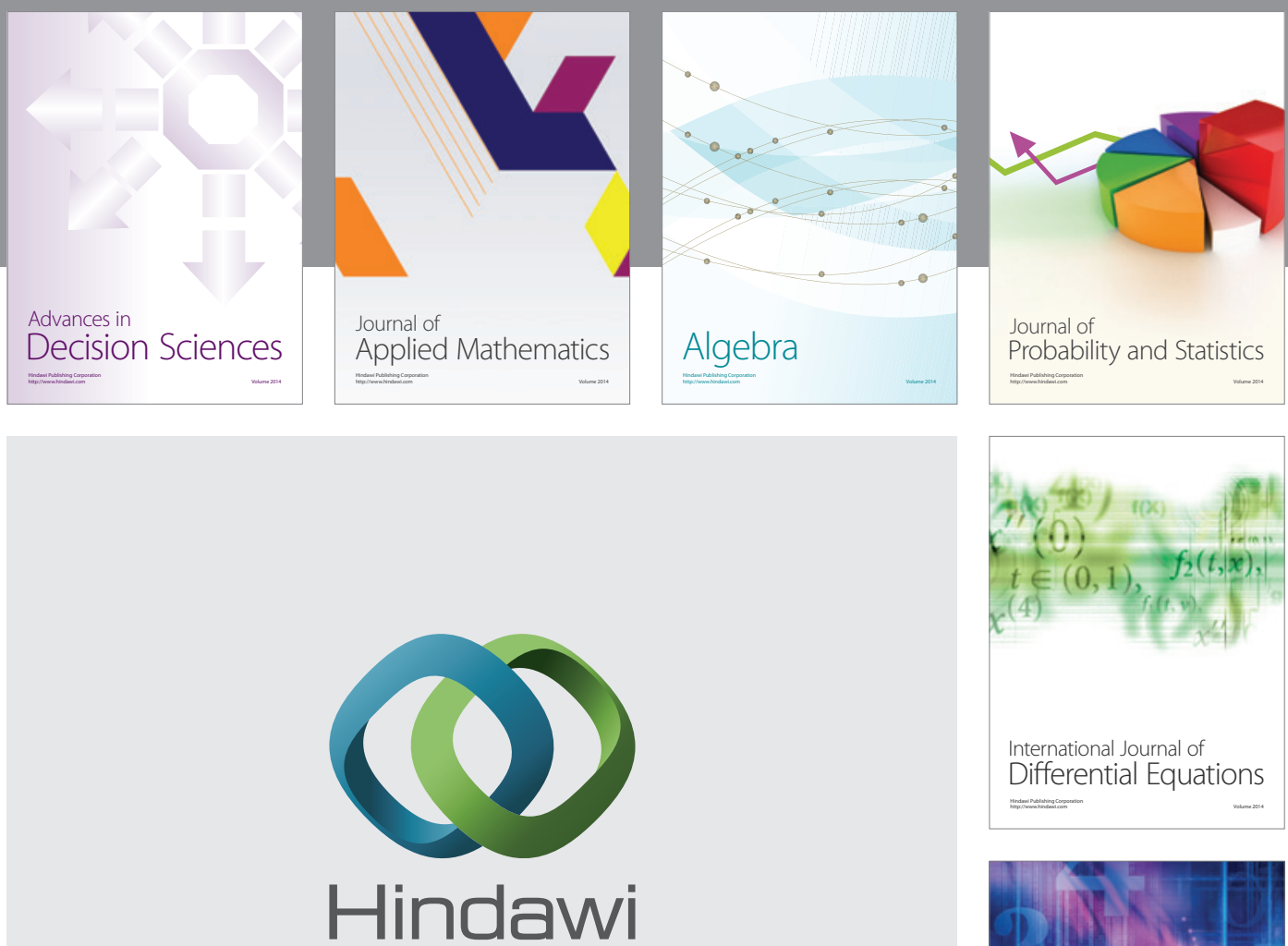

Submit your manuscripts at http://www.hindawi.com
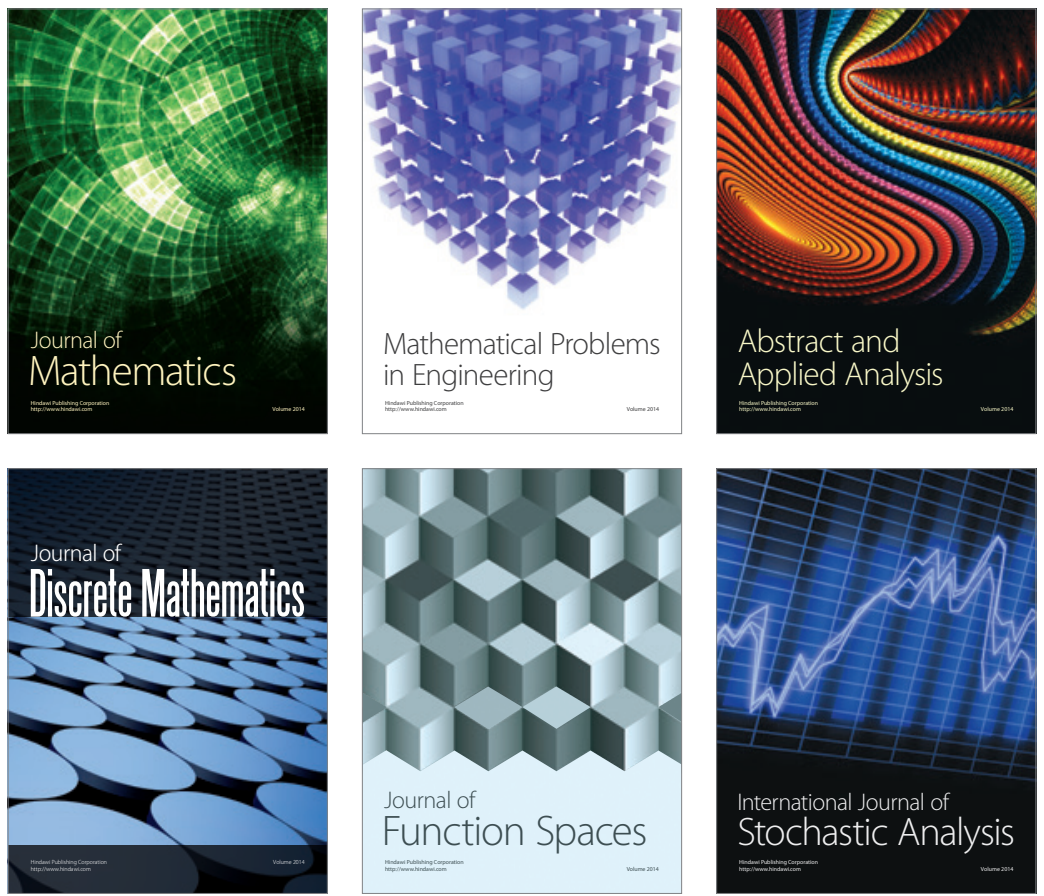

Journal of

Function Spaces

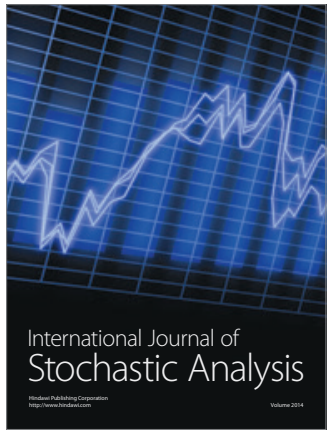

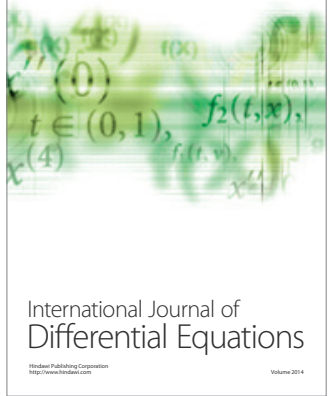
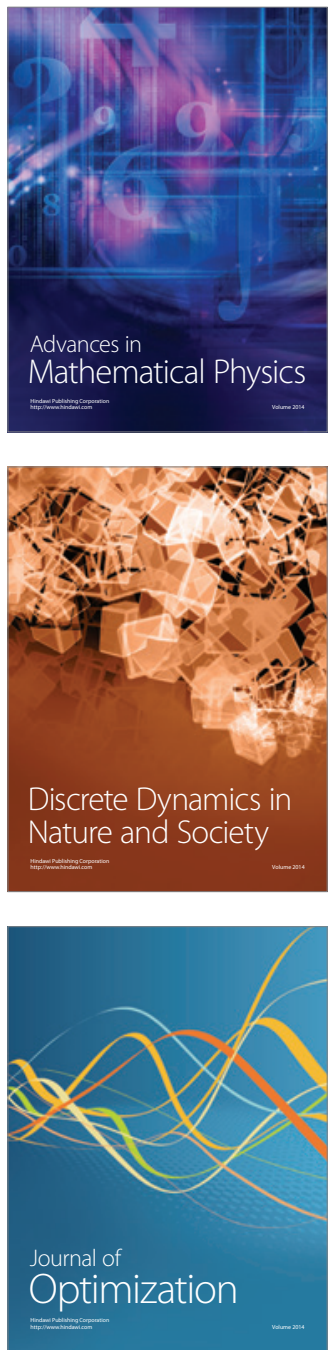